\title{
Patient Flow und optimierter Ressourceneinsatz
}

\author{
Die intransparenten und unsteten Patientenströme in der klinikgebundenen Notfallversorgung führen \\ aktuell zu mitunter sehr langen Wartezeiten und verzögerter Patientenversorgung. Frühzeitige Patienten- \\ klassifikation durch eine möglichst zeitnahe Triage ermöglicht eine Optimierung der Patientenversorgung \\ und des erforderlichen Personaleinsatzes. Die zentrale Notfallaufnahme fungiert als effektiver Stellhebel \\ für die bedarfsgerechte Steuerung von Akutpatientenströmen.
}

Die Versorgung akuter Patienten erfolgt aktuell über variierende und multidisziplinäre Versorgungsketten, die zum Teil geprägt sind durch Intransparenz, Prozessbrüche, Kommunikationsdefizite und Informationslücken. Hieraus ergeben sich mitunter lebensbedrohliche Zeitverzögerungen, Fehlversorgungen oder Behandlungsfehler, die zu einer suboptimalen Versorgungsqualität führen. Ferner treffen derartige Abstimmungsmängel auf knappe Verfügbarkeit personeller Ressourcen in der übergreifenden ambulanten und stationären Notfallversorgung.

Andererseits lassen sich zunehmend ausdifferenzierte Patientensegmente (z.B. Polytrauma-, Schock-, Schlaganfall-, Herzinfarkt- sowie geriatrischen Patienten) identifizieren, die eine jeweilig spezifizierte sowie adaptierte Versorgungskette und Teamqualifikationen erfordern. Aufgrund steigender Patientenzahlen sowie der Bedeutung der Notaufnahme für die nachfolgenden Versorgungsprozesse gewinnt die Notaufnahme in den letzten Jahren verstärkt an qualitätsbezogener, ökonomischer und strategischer Relevanz.

\section{Vernetzung und Triage}

Die bisherigen Versorgungsstrukturen und -prozesse in der Notfallversorgung von Krankenhäusern sind zunehmend gekennzeichnet durch eigenständige, zentrale und interdisziplinärer Notaufnahmen. Die Zentrale Notaufnahme (ZNA) fungiert in der Regel auch als Zentrale Patientenaufnahme (ZPA) und ist somit nicht nur die interdisziplinäre Erstanlaufstelle für Patienten im Krankenhaus, sondern damit auch Dreh- und Angelpunkt für die weiteren patientenbezogenen Versorgungsleistungen im und außerhalb des Krankenhauses. Die ZNA/ ZPA bündelt interprofessionell und fachübergreifend die für die Statusermittlung erforderlichen räumlichen, personellen, technischen und materiellen Ressourcen. Ferner bildet sie die wesentliche Kommunikations- und Schnittstelle zu den externen Versorgungs- und Kooperationspartnern (zum Beispiel Leitstelle, Rettungsdienst, andere Krankenhäuser, Hausärzte). Zur frühzeitigen und patientenbezogenen Steuerung der Behandlungskapazitäten ist es erforderlich, die Krankheitsschwere der Patienten einzuschätzen und die Behandlungsabfolgen mittels Triage frühestmöglich zu priorisieren und festzulegen.

\section{Transparenz und Patientenlogistik}

Die Patientenbetreuung erfordert einen konzeptionellen Patienten Flow (Patientenfluss), vom Eintreffen bis zum Übergang des Patienten in eine weiterführende Versorgungsstufe. Der Patientenfluss umfasst neben medizinisch-pflegerischen, administrativen und serviceorientierten Aspekten auch die Einbeziehung vor- und nachgelagerter Anforderungen und Aktivitäten sowie das Patientenverhalten. Als übergreifende Organisations- und Optimierungsansätze bieten sich unter anderem die Schaffung einer ausreichenden Transparenz sowie die Koordination einer abgestimmten Patientenlogistik an.

Transparenz bildet in komplexen Systemen, neben einer intensivierten Kommunikation, stets den ersten Ansatz zur Verbesserung. Transparenz schafft neben zugänglichen
Informationen und verständlicher Nachvollziehbarkeit über Abläufe, Sachverhalte, Vorhaben sowie Verhaltens- und Entscheidungsprozesse, insbesondere Vertrauen zwischen den beteiligten Akteuren. Die Patientenlogistik umfasst dabei die begleitete und nicht begleitete Bewegung und Unterbringung von ambulanten und stationären Patienten innerhalb eines Krankenhauses und eröffnet dadurch die Möglichkeit der Verbesserung der Versorgungsqualität und des Ressourceneinsatzes im Krankenhaus. Der Patient muss dabei zielgerichtet informiert, gesteuert und koordiniert werden, beispielsweise mittels Case Management.

\section{Patientenklassifikation und Versorgungsschemata}

Hinsichtlich einer patientenbezogenen Steuerung und Koordination der Versorgungsprozesse sowie der dafür erforderlichen Personal- und Ressourceneinsätze (z. B. qualifiziertes Personal, Patienteninformationen und Know-how, räumliche und diagnostische Möglichkeiten) ist es erforderlich, die prognostizierten und real auftretenden Patientengruppen zu klassifizieren, zum Beispiel Grippewelle, Suchtmittelmissbrauch, Selbsteinweiser usw. Hierbei gilt es, auf der Basis verfügbarer und erforderlicher patientenbezogener Daten und Informationen,eintreffende Patienten aussagekräftigen Patientensegmenten zuzuordnen. So können zum Beispiel Vitalparameter, Indikationen oder Komplikationen genutzt werden für die Zuordnung der Patienten als Polytraumaoder Infarktpatient oder für die Einordnung als Bagatellfall. 
Die resultierenden aggregierten Informationen (z. B. Patientenklassifikationssystem, Injury Severity Score) präsentieren verdichtete Daten, auf deren Basis die unterschiedlichen Versorgungsprozesse (zum Beispiel Anamnese, Erstversorgung, Diagnostik, stationäre Aufnahme) geplant, gesteuert, durchgeführt und dokumentiert werden können. Zur übersichtlichen und leicht zugänglichen Unterstützung der patientensegmentbezogenen Versorgung in der zentralen Notfallversorgung bieten sich spezifische Versorgungsschemata an, zum Beispiel ABCDE-Schema und Behandlungsleitlinien. Diese ermöglichen, mittels bestimmter Ordnung und festgelegtem Vorgehen, ein einfaches konzeptionelles Schema, nach dem sich die Versorgung orientieren sollte. Durch die grafische und zeichnerische Darstellung werden die Situationsbeurteilung sowie die auszuführende Versorgung unterstützt.

\section{Polytrauma- und Schockraum- versorgung}

Polytraumapatienten sind per Definition Patienten mit mehreren gleichzeitig erlittenen Verletzungen, von denen mindestens eine oder die Summe aller Verletzungen lebensbedrohlich sind. In der Regel entstehen diese Verletzungen unfallbedingt und die Patienten müssen im Schockraum versorgt werden. Neben lebensrettenden Sofortmaßnahmen (z. B. Sicherung der Atemwege, Blutstillung und Absicherung bei drohendem Querschnittes) erfolgt hier die weitere Erstversorgung, -anamnese und -diagnostik (z. B. Traumaspirale) sowie die Initiierung weiterführende spezieller Diagnostik- und Therapie (z. B. Intensivstation, OP, Spezialklinik).

In der ZNA des St. Willibrord-Spitals Emmerich wurden im Bereich der zentralen Notfallversorgung u.a. konkrete Versorgungsschemata bezüglich der interdisziplinären Versorgung von Polytrauma- und Schockpatienten bzw. Reanimationen erarbeitet, visualisiert und etabliert. Diese sind speziell auf die interprofessionellen und örtlichen Gegebenheiten zugeschnitten. Neben der Vorbereitung (z. B. Bereitstellung röntgendurchlässiger Liege/Defibrillator) bei ange-

\section{Die unsteten Patientenströme in der stationären Notfall- und Akut- versorgung führen aktuell zu wach- senden Wartezeiten und verzögerter Notfallversorgung.}

kündigten Patienten (z. B. durch Leitstelle, Pforte) sowie der aktivierten ständigen Leistungsbereitschaft (z. B. unangekündigter Patient) umfasst das Versorgungsschemata die abgestufte Vorgehensweise der medizinisch-pflegerischen Versorgung (z.B. Erfassung der Vitalparameter, Reanimationsmaßnahmen, Sonografie Abdomen und Thorax) durch das Basisteam (z. B. DGKP, MFA, MTA, Chirurg, Internistin) bzw. das erweiterte Team (z. B. Intensivpfleger, Anästhesist, Kardiologe). Ferner werden die erforderlichen organisatorischen und administrativen Aufgaben (z. B. Erfassung Personalien, Protokollerstellung, Verlegungsbericht, Nachbesprechung) sowie die Optionen und Anforderungen der Weiterleitung bzw. Verlegung des Patienten dargestellt. Hierdurch werden die intuitiven und gelebten Versorgungsaktivitäten mit den evidenten Standards der jeweiligen Notfallversorgung verknüpft. Dies führt sowohl zu einer evidenzbasierten Notfallund Akutversorgung als auch zu einer qualitativen Patientenorientierung und einem optimierten Ressourceneinsatz.

\section{Übergreifende Koordination der Patientenversorgung}

Steigende Patientenzahlen, erweiterte diagnostische Verfahren oder ausgedehnte Tätigkeitsbereiche führen auch in der Notfallversorgung zu einer verstärkten Arbeitsverdichtung, der nur bedingt durch veränderten Personalbestand begegnet werden kann. Vielmehr ist es erforderlich, neue Ansätze der Versorgung sowie der damit verbundenen Kommunikation, Koordination und Kooperation zwischen den involvierten Akteuren, zu entwickeln, zu er- proben und zu evaluieren. Mögliche Ansätze sind hier u.a. Schockraummanagement, Patientenklassifikationssystem, interdisziplinäres Kommunikationskonzept, soziale Kompetenzen der Health Professionals. Ziel ist es, die ZNA und ZPA zu einem Knotenpunkt der vernetzten und übergreifenden Patientenbehandlung in der integrierten Versorgung zu entwickeln.

Neben der Entzerrung bestehender Patientenstaus, zum Beispiel durch digitale Portalpraxen und der frühzeitigen Anamnese relevanter diagnostischer und patientenbezogener Indikatoren (zum Beispiel durch Telefon-Triage und Ersteinschätzung) gilt es, die individuelle Versorgungsqualität über Sektoren-, Organisations- und Professionsgrenzen hinweg abgestimmt und optimiert zu verbessern und zu gewährleisten. Hieraus ergibt sich die Notwendigkeit gleichermaßen für Entscheidungs- und Patientenverantwortliche im Krankenhaus entsprechende Strategien zu entwickeln, Konzepte zu etablieren und Kulturen zu unterstützen, die die patientenbezogene (Notfall-)Versorgung im Krankenhaus gestalten, steuern und erbringen.

\section{- Weiterführende Literatur:}

Francke A et al. Interdisziplinäre Notaufnahme: Ein Ratgeber für Aufnahme und Bereitschaftsdienst. Stuttgart, Thieme, 2009 Kaschwich M, Seekamp A. Triage in der Notaufnahme. Notf.med. up2date 2011; 6: 189-202

Schmidt CM et al. Notfallversorgung in Deutschland. Essen, RWI, 2016

Dr. Nanni Reckwitz, Oberärztin der Zentralen Notaufnahme, Fachärztin für Allgemeinmedizin/Palliativmedizin, St. Willibrord Spital EmmerichRees gGmbH

Sybille Reckwitz, Auszubildende der Gesundheits- und Krankenpflege, St. Willibrord Spital Emmerich-Rees gGmbH, Bildungszentrum Niederrhein Wesel

Marcel Thra, M.A., Advanced Nurse Practitioner, St. Willibrord Spital Emmerich-Rees gGmbH 